\title{
A TCAS-II Resolution Advisory Detection Algorithm
}

\author{
César Muñoz* Anthony Narkawicz \\ James Chamberlain \\ NASA, Langley Research Center, Hampton, VA, 23681, USA
}

The Traffic Alert and Collision Avoidance System (TCAS) is a family of airborne systems designed to reduce the risk of mid-air collisions between aircraft. TCAS II, the current generation of TCAS devices, provides resolution advisories that direct pilots to maintain or increase vertical separation when aircraft distance and time parameters are beyond designed system thresholds. This paper presents a mathematical model of the TCAS II Resolution Advisory (RA) logic that assumes accurate aircraft state information. Based on this model, an algorithm for RA detection is also presented. This algorithm is analogous to a conflict detection algorithm, but instead of predicting loss of separation, it predicts resolution advisories. It has been formally verified that for a kinematic model of aircraft trajectories, this algorithm completely and correctly characterizes all encounter geometries between two aircraft that lead to a resolution advisory within a given lookahead time interval. The RA detection algorithm proposed in this paper is a fundamental component of a NASA sense and avoid concept for the integration of Unmanned Aircraft Systems in civil airspace.

\section{Nomenclature}

$\mathbf{s}_{o} \quad$ Current horizontal component of ownship's position

$s_{o z} \quad$ Current ownship's altitude

$\mathbf{v}_{o} \quad$ Current horizontal component of ownship's velocity

$v_{o z} \quad$ Current ownship's vertical speed

$\mathbf{s}_{i} \quad$ Current horizontal component of intruder's position

$s_{i z} \quad$ Current intruder's altitude

$\mathbf{v}_{i} \quad$ Current horizontal component of intruder's velocity

$v_{i z} \quad$ Current intruder's vertical speed

$t_{\text {cpa }} \quad$ Time to closest horizontal point of approach

$t_{\text {coa }} \quad$ Time to co-altitude

$\tau \quad$ Current ownship's tau

$\tau_{\bmod \ell} \quad$ Current ownship's modified tau for sensitivity level $\ell$

$\mathrm{TAU}_{\ell} \quad \mathrm{RA}$ tau threshold for sensitivity level $\ell$

$\mathrm{DMOD}_{\ell} \quad \mathrm{RA}$ DMOD for sensitivity level $\ell$

ZTHR $_{\ell} \quad \mathrm{RA}$ vertical threshold for sensitivity level $\ell$

$\mathrm{HMD}_{\ell} \quad \mathrm{RA}$ Horizontal Miss Distance for sensitivity level $\ell$

$\mathrm{ALIM}_{\ell} \quad \mathrm{RA}$ altitude limit for sensitivity level $\ell$

\section{Subscript}

o Ownship information

$i \quad$ Intruder information

$\ell \quad$ Current ownship's sensitivity level

$x \quad$ Northern component of a position or velocity vector

$y \quad$ Eastern component of a position or velocity vector

\footnotetext{
${ }^{*}$ Research Computer Scientist, AIAA Member.
} 


\section{Introduction}

The Unmanned Aircraft Systems (UAS) Integration in the National Airspace System (NAS) is a NASA research project that addresses the integration of civil UAS into non-segregated airspace operations. One of the major challenges of this integration is the lack of an on-board pilot to comply with the legal requirement identified in the US Code of Federal Regulations (CFR) that pilots see and avoid other aircraft in their vicinity. As a means of compliance with this legal requirement, the final report of the FAA-sponsored Sense and Avoid (SAA) Workshop [1] defines the concept of sense and avoid for remote pilots as "the capability of a UAS to remain well clear from and avoid collisions with other airborne traffic." Hence, collision avoidance is a critical element of any sense and avoid concept for the integration of UAS in the NAS.

The Traffic Alert and Collision Avoidance System (TCAS) is a family of airborne devices that are designed to reduce the risk of mid-air collisions between aircraft equipped with operating transponders [2]. TCAS has evolved through extensive development and a number of versions since its initial operational evaluation in 1982. TCAS II, the current generation of TCAS devices, is mandated in the US for aircraft with greater than 30 seats or a maximum takeoff weight greater than 33,000 pounds. Although it is not required, TCAS II is also installed on many turbine-powered general aviation aircraft. Version 7.0 is the current operationallymandated version of TCAS II, and Version 7.1 has been standardized [3].

In contrast to TCAS I, the first generation of TCAS devices, TCAS II provides resolution advisories (RAs). RAs are visual and vocalized alerts that direct pilots to maintain or increase vertical separation with intruders that are considered collision threats. TCAS II resolution advisories can be corrective or preventive depending on whether the pilot is expected to change or maintain the aircraft's current vertical speed. Corrective RAs are particularly disruptive to the air traffic system since they may cause drastic evasive maneuvers. For this reason, they are intended as a last resort maneuver when all other means of separation have failed.

The Sense and Avoid (SAA) concept for Unmanned Aircraft Systems (UAS) described in [4] rests on interoperability principles that take into account both the Air Traffic Control (ATC) environment as well as existing systems such as TCAS. Specifically, the concept addresses the determination of well clear values that are large enough to avoid issuance of TCAS II resolution advisories. It relies on airborne and ground capabilities that predict encounter geometries that will cause an RA. These capabilities allow UAS pilots to take non-disruptive preventive actions early enough to avoid issuance of corrective RAs. The main contribution of this paper is an algorithm for TCAS II RA detection that can be used to implement those SSA airborne and ground capabilities.

The RA detection algorithm presented in this paper is based on a mathematical model of the TCAS II resolution advisory logic that assumes accurate vector state information for two aircraft. It is analogous to a conflict detection algorithm but instead of predicting loss of separation, it predicts RAs. It has been formally verified in the Program Verification System (PVS) [5] that assuming aircraft linear trajectories, the algorithm completely and correctly characterizes all encounter geometries that will cause an RA within a given lookahead time interval. The formal development presented in this paper is part of the NASA's Airborne Coordinated Resolution and Detection (ACCoRD) mathematical framework, which is electronically available from http://shemesh.larc.nasa.gov/people/cam/ACCoRD.

The rest of this paper is organized as follows. Section II provides a high level description of the TCAS II resolution advisory logic. A mathematical model of this logic is presented in Section III. Section IV proposes an algorithm for resolution advisory detection and states its main correctness property. Section V presents an algorithm that checks whether a given RA is corrective or preventive. Section VI discusses related work. The last section concludes the paper.

\section{TCAS II Resolution Advisory Logic}

TCAS consists of several hardware and software sub-systems. These sub-systems provides means for limited surveillance and communication, and perform tasks such as traffic identification, tracking, and collision avoidance. This paper focuses on the collision detection sub-system that deals with resolution advisories.

TCAS collision detection logic uses the concept of $\operatorname{tau}(\tau)$ to estimate the time to closest point of approach (CPA) between the ownship and one given traffic aircraft known as intruder. The time tau is defined as 
range $(r)$ over closure rate, where closure rate is the negative of the range rate $(\dot{r})$, i.e.,

$$
\tau \equiv-\frac{r}{\dot{r}},
$$

Both range and range rate are derived from TCAS interrogations of the intruder's transponder, nominally at one-second intervals. The time tau and the actual time to CPA coincide only when the aircraft are on a perfect collision course and not accelerating. If the aircraft will merely pass near each other, then tau is only an approximation of time to CPA. In this case, tau will decrease to a minimum value shortly before actual CPA and then sharply increase until CPA, at which time the value of tau is undefined.

In the vertical dimension, time to co-altitude and vertical separation are used instead of tau and range. Time to co-altitude is sometimes called vertical tau and is computed as vertical separation divided by vertical closure rate. In the general case, a resolution advisory is issued when range and vertical separation are below horizontal and vertical distance thresholds called DMOD and ZTHR, respectively, and tau and time to coaltitude are below a time threshold called TAU. Since the ratio of range and range rate tends to be lower at closer distances, the minimum value that tau will attain in the near future is a time that varies directly with the nearness of the encounter. This property of tau means that the selection of a time threshold value, i.e., TAU, at which to alert for a collision threat determines not only the time to react to the threat, but also the size of protected airspace within which a given threat encounter will cause an alert.

An effective TCAS logic requires a tradeoff between necessary protection and unnecessary advisories. This tradeoff is partly managed by controlling the sensitivity level (SL), which varies with the altitude of the ownship. Higher SLs are selected for higher altitudes, where generally speeds are higher and separations are larger. Among other things the SL controls the tau thresholds for RA issuance, and therefore the dimensions of protected airspace around each TCAS-equipped aircraft.

Two problems may arise with use of the simple definition of tau given by Formula (1). The first problem involves threat encounters with low range closure rates, and the second problem involves high closure rates with large miss distances. TCAS II addresses the low-closure rate problem by using a modified definition of tau [3]:

$$
\tau_{\text {mod }} \equiv-\frac{r^{2}-\mathrm{DMOD}^{2}}{r \dot{r}} .
$$

DMOD was designed to provide approximately an RA-threshold amount of reaction time for an intruder that accelerated toward the ownship at a sustained $1 / 3 \mathrm{~g}[6]$. Modified tau values are nearly identical to the true value of tau at large ranges and range rates but are smaller, i.e., more conservative, for smaller ranges and rates. Formula (2) assumes that the closure rate is not zero and that the current range is greater or equal than DMOD.

TCAS Versions 7.0 and higher address the high-closure-rate, nuisance-RA problem by employing a horizontal miss distance (HMD) filter [7]. The HMD filter employs a parabolic range tracker to provide projected range acceleration as well as projected range and range rate, and uses the range acceleration to detect horizontal miss distances that are sufficiently large so as not to be a collision threat (range acceleration will be zero for non-accelerating aircraft on a collision course, but will be positive if the encounter has a miss distance). The HMD filter employs numerous noise filters and maneuver checks whose explanations are beyond the scope of this paper, but the end result is that the filter will suppress RA issuances for horizontal miss distances at CPA that are approximately equal to or greater than the DMOD values.

\begin{tabular}{|c|c|c|c|c|c|c|}
\hline $\begin{array}{l}\text { Ownship Altitude } \\
\text { (feet) }\end{array}$ & SL & $\begin{array}{l}\text { TAU } \\
(\mathrm{sec})\end{array}$ & $\begin{array}{c}\text { DMOD } \\
\text { (nmi) }\end{array}$ & $\begin{array}{c}\text { ZTHR } \\
\text { (feet) }\end{array}$ & $\begin{array}{c}\text { ALIM } \\
\text { (feet) }\end{array}$ & $\begin{array}{r}\text { HMD } \\
(\mathrm{nmi})\end{array}$ \\
\hline $1000-2350$ & 3 & 15 & 0.20 & 600 & 300 & 0.4 \\
\hline $2350-5000$ & 4 & 20 & 0.35 & 600 & 300 & 0.57 \\
\hline $5000-10000$ & 5 & 25 & 0.55 & 600 & 350 & 0.74 \\
\hline $10000-20000$ & 6 & 30 & 0.80 & 600 & 400 & 0.82 \\
\hline $20000-42000$ & 7 & 35 & 1.10 & 700 & 600 & 0.98 \\
\hline$>42000$ & 7 & 35 & 1.10 & 800 & 700 & 0.98 \\
\hline
\end{tabular}

Table 1. TCAS Sensitivity Level Definition and Alarm Thresholds for RAs 
Table 1 shows the altitude bands for each SL and the associated thresholds for RA issuance $[2,7]$. The distance threshold value ALIM is used to determine if a particular RA is corrective or preventive. For example, when a TCAS-equipped aircraft is between 20000 and 42000 feet (SL 7), the tau threshold for RA issuance is 35 seconds, and generally an RA will be issued if both horizontal and vertical tau fall below this value. An RA will also be issued for low vertical rate encounters if the current altitude difference is less than the vertical threshold (ZTHR) value of 700 feet. Once TCAS determines that an RA is required, it must determine the type of RA needed. In order to do this, TCAS estimates the altitude difference at CPA for various RA types; if the altitude difference will be less than the altitude limit (ALIM) value (600 feet in this example), then the RA will be corrective (e.g., "Climb" if level), requiring a trajectory change to regain at least ALIM feet of vertical separation; otherwise the RA will be preventive (e.g., "Dont Descend" if level), requiring no trajectory change.

The next section presents a mathematical model of the TCAS II RA logic that formalizes the description provided above.

\section{Vectorized Model of the TCAS II Resolution Advisory Logic}

While TCAS II uses different mechanisms to track aircraft as accurately as possible, the mathematical model presented in this section assumes that accurate aircraft surveillance information is available as horizontal and vertical components in a 3-dimensional airspace. Through out this paper, letters in bold-face denote 2-D vectors. Vector operations such as addition, subtraction, scalar multiplication, dot product, i.e., $\mathbf{s} \cdot \mathbf{v} \equiv s_{x} v_{x}+s_{y} v_{y}$, and the norm of a vector, i.e., $\|\mathbf{s}\| \equiv \sqrt{\mathbf{s} \cdot \mathbf{s}}$, are defined in a 2-D Euclidean geometry. The expression $\mathbf{v}^{\perp}$ denotes the 2 -D right perpendicular of $\mathbf{v}$, i.e., $\left(v_{y},-v_{x}\right)$. Furthermore, the function root, defined by Formula (3), computes the roots of the quadratic equation $a x^{2}+b^{x}+c=0$. For completeness, it is defined such that it returns the value 0 when the roots are undefined. In this paper, the values returned by root are only used in a context where $a \neq 0$ and $b^{2}-4 a c \geq 0$.

$$
\operatorname{root}(a, b, c, \epsilon) \equiv \begin{cases}\frac{-b+\epsilon \sqrt{b^{2}-4 a c}}{2 a} & \text { if } a \neq 0 \text { and } b^{2}-4 a c \geq 0, \\ 0 & \text { otherwise. }\end{cases}
$$

Assuming constant velocities, the horizontal positions of the ownship and intruder aircraft at a time $t \geq 0$, are given by

$$
\begin{aligned}
\mathbf{s}_{o}(t) & \equiv \mathbf{s}_{o}+t \mathbf{v}_{o}, \\
\mathbf{s}_{i}(t) & \equiv \mathbf{s}_{i}+t \mathbf{v}_{i},
\end{aligned}
$$

respectively. As it simplifies the mathematical development, some definitions in this paper use a relative coordinate system where the intruder is static at the center of the system. In this relative system, the ownship is located at $\mathbf{s}=\mathbf{s}_{o}-\mathbf{s}_{i}$ and moves at relative velocity $\mathbf{v}=\mathbf{v}_{o}-\mathbf{v}_{i}$. Therefore, the relative horizontal position of the ownship with respect to the traffic aircraft at any time $t$ can be defined as follows.

$$
\mathbf{s}(t) \equiv \mathbf{s}+t \mathbf{v}
$$

The range between the aircraft at any time $t$ is given by ${ }^{\mathrm{a}}$

$$
r(t) \equiv\|\mathbf{s}(t)\|=\sqrt{\|\mathbf{s}\|^{2}+2 t \mathbf{s} \cdot \mathbf{v}+t^{2}\|\mathbf{v}\|^{2}}
$$

Closure rate is the derivative of $r(t)$ with respect to $t$, i.e.,

$$
\dot{r}(t) \equiv \frac{\mathbf{s} \cdot \mathbf{v}+t\|\mathbf{v}\|^{2}}{\|\mathbf{s}(t)\|} .
$$

Given a relative position $\mathbf{s}$ and velocity $\mathbf{v}$, the time of horizontal closest point of approach, denoted $t_{\text {cpa }}$, is the time $t$ that satisfies $\dot{r}(t)=0$. Hence,

$$
t_{\mathrm{cpa}}(\mathbf{s}, \mathbf{v}) \equiv-\frac{\mathbf{s} \cdot \mathbf{v}}{\|\mathbf{v}\|^{2}}
$$

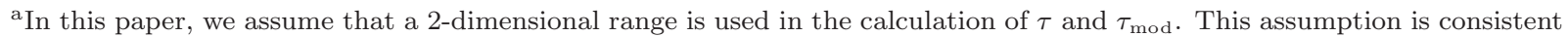
with the use of these values in horizontal threshold checks done by the TCAS II resolution advisory logic. 
By convention, $t_{\mathrm{cpa}}$ is defined as 0 when the velocities of the ownship and intruder aircraft are identical, i.e., $t_{\mathrm{cpa}}(\mathbf{s}, \mathbf{v})=0$, if $\|\mathbf{v}\|^{2}=0$.

Given a relative position $\mathbf{s}$ and velocity $\mathbf{v}$, Formula (1) and Formula (2) can be written in a vector form as follows.

$$
\begin{gathered}
\tau(\mathbf{s}, \mathbf{v}) \equiv-\frac{r}{\dot{r}}=-\frac{r(0)}{r(0)}=-\frac{\|\mathbf{s}\|}{\frac{\mathbf{s} \cdot \mathbf{v}}{\|\mathbf{s}\|}}=-\frac{\|\mathbf{s}\|^{2}}{\mathbf{s} \cdot \mathbf{v}}, \\
\tau_{\bmod \ell}(\mathbf{s}, \mathbf{v}) \equiv \frac{\operatorname{DMOD}_{\ell}^{2}-\|\mathbf{s}\|^{2}}{\mathbf{s} \cdot \mathbf{v}} .
\end{gathered}
$$

Formula (1) and Formula (2) are undefined when the closure rate is 0. This happens when both aircraft have the same velocity vector, i.e., same direction and same speed, and it also happens when at current time the aircraft are at the closest point of approach. In the vector form of these formulas, i.e., Formula (10) and Formula (11), the singularity is equivalently expressed as $\mathbf{s} \cdot \mathbf{v}=0$. The dot product $\mathbf{s} \cdot \mathbf{v}$ also characterizes whether the aircraft are horizontally diverging, i.e., $\mathbf{s} \cdot \mathbf{v}>0$, or horizontally converging, i.e., $\mathbf{s} \cdot \mathbf{v}<0$. It can be seen from Formula (10) that when the aircraft are converging, $\tau$ is positive. In the TCAS II RA logic, Formula (11) is used in a context where the aircraft are horizontally converging. In that case, modified tau, i.e., $\tau_{\bmod \ell}$, is positive when the current range is greater than $\mathrm{DMOD}_{\ell}$.

As explained in Section II, the TCAS II RA logic checks the value of $\tau_{\text {mod }}$ against horizontal and vertical thresholds to determine if a resolution advisory will be issued. These checks can be mathematically modeled using the functions Horizontal $\mathrm{RA}_{\ell}$ and Vertical $\mathrm{RA}_{\ell}$ below.

The function Horizontal_RA $A_{\ell}$ takes as parameters the relative horizontal position $\mathbf{s}$ and velocity $\mathbf{v}$ of the aircraft. It returns true if, for the given input and sensitivity level $\ell$, the horizontal thresholds are satisfied.

$$
\text { Horizontal_RA }{ }_{\ell}(\mathbf{s}, \mathbf{v}) \equiv\|\mathbf{s}\| \leq \operatorname{DMOD}_{\ell} \text { or }\left(\mathbf{s} \cdot \mathbf{v}<0 \text { and } \tau_{\bmod \ell}(\mathbf{s}, \mathbf{v}) \leq \mathrm{TAU}_{\ell}\right) \text {. }
$$

In order to model the vertical check performed by the TCAS II RA logic, it is necessary to define the time to co-altitude $t_{\mathrm{coa}}$. This time satisfies $s_{z}+t_{\mathrm{coa}} v_{z}=0$, where $s_{z} \equiv s_{o z}-s_{i z}$ and $v_{z} \equiv v_{o z}-v_{i z}$. Therefore, for a given vertical separation $s_{z}$ and relative non-zero vertical speed $v_{z}$,

$$
t_{\text {coa }}\left(s_{z}, v_{z}\right) \equiv-\frac{s_{z}}{v_{z}}
$$

Similar to the horizontal case, the product $s_{z} v_{z}$ characterizes whether the aircraft are vertically diverging, i.e., $s_{z} v_{z}>0$, or vertically converging, i.e., $s_{s} v_{z}<0$. In the TCAS II RA logic, Formula (13) is used in a context where the aircraft are vertically converging. In this case, $t_{\text {coa }}$ is always positive. The function Vertical_RA $\ell$, which returns true when the vertical thresholds are satisfied for a sensitivity level $\ell$, is defined as follows.

$$
\text { Vertical } \mathrm{RA}_{\ell}\left(s_{z}, v_{z}\right) \equiv\left|s_{z}\right| \leq \mathrm{ZTHR}_{\ell} \text { or }\left(s_{z} v_{z}<0 \text { and } t_{\text {coa }}\left(s_{z}, v_{z}\right) \leq \mathrm{TAU}_{\ell}\right) .
$$

where $s_{z}$ and $v_{z}$ are, respectively, the vertical separation and the relative vertical velocity of the aircraft.

In addition to the horizontal and vertical checks, TCAS Version 7.0 introduces a filter that inhibits resolution advisories when the projected point of closest approach is larger than a given horizontal miss distance. In the formal model presented in this paper, this functionality is accomplished by a state-based 2-D conflict detection algorithm called $\mathrm{CD}_{2} \mathrm{D}_{\infty}$.

The function $\mathrm{CD}_{2} \mathrm{D}_{\infty}$ takes as parameters the relative state of the aircraft, i.e., relative position $\mathbf{s}$ and relative velocity $\mathbf{v}$, a minimum separation distance $D>0$ and a time $B \geq 0$. It returns a Boolean value that indicates whether or not the aircraft will be within horizontal distance $D$ of one another at any time after $B$ along trajectories which are linear projections of their current states.

$$
\begin{aligned}
\mathrm{CD}_{2} \mathrm{D}_{\infty}(\mathbf{s}, \mathbf{v}, D, B) \equiv & (\|\mathbf{v}\|=0 \text { and }\|\mathbf{s}\| \leq D) \text { or } \\
& (\|\mathbf{v}\|>0 \text { and } \Delta(\mathbf{s}, \mathbf{v}, D) \geq 0 \text { and } \Theta(\mathbf{s}, \mathbf{v}, D, 1) \geq B),
\end{aligned}
$$

where

$$
\begin{aligned}
\Delta(\mathbf{s}, \mathbf{v}, D) & \equiv D^{2}\|\mathbf{v}\|^{2}-\mathbf{s} \cdot \mathbf{v}^{\perp}, \text { and } \\
\Theta(\mathbf{s}, \mathbf{v}, D, \epsilon) & \equiv \operatorname{root}\left(\|\mathbf{v}\|^{2}, 2(\mathbf{s} \cdot \mathbf{v}),\|\mathbf{s}\|^{2}-D^{2}, \epsilon\right) .
\end{aligned}
$$


When $\Delta(\mathbf{s}, \mathbf{v}, D) \geq 0$, the function $\Theta$ computes the times when the aircraft will loss separation, if $\epsilon=-1$, or regain separation, if $\epsilon=1$.

It has been formally proved in the PVS theorem prover that the function $\mathrm{CD}_{2} \mathrm{D}_{\infty}$ completely and correctly predicts violations of a minimum distance $D$ after time $B$, i.e., the following statement holds.

Proposition 1. For all vectors $\mathbf{s}=\mathbf{s}_{o}-\mathbf{s}_{i}, \mathbf{v}=\mathbf{v}_{o}-\mathbf{v}_{i}$, distance $D>0$, and time $B \geq 0, C D 2 D_{\infty}(\mathbf{s}, \mathbf{v}, D, B)$ returns true if and only if there exists a time $t \geq B$ where $\|\mathbf{s}+t \mathbf{v}\|<D$.

Using Horizontal_RA $A_{\ell}$, Vertical_RA, and $C D 2 D_{\infty}$, the function that determines whether or not an RA will be issued for the ownship can be defined as follows.

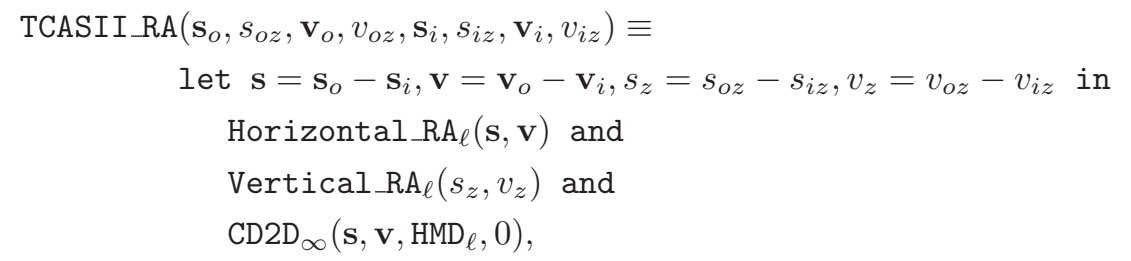

where $\ell$ is the sensitivity level corresponding to $s_{o z}$ and $\mathrm{HMD}_{\ell}$ is the horizontal miss distance for that sensitivity level. Furthermore, the function that checks if an RA will be issued for the ownship at a given future time $t<t_{\text {cpa }}(\mathbf{s}, \mathbf{v})$ can be defined as follows. ${ }^{\mathrm{b}}$

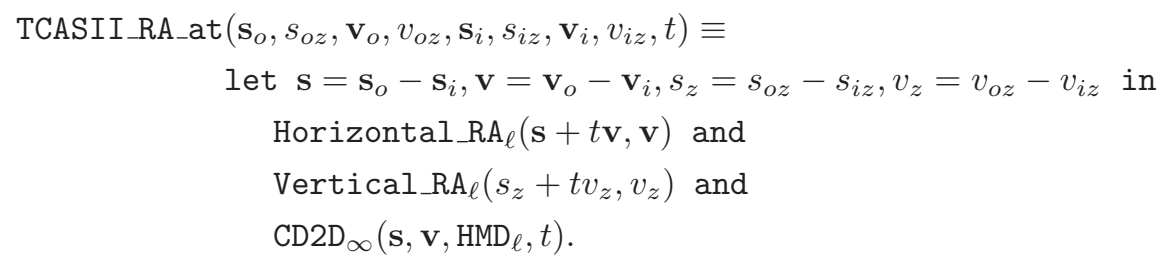

\section{Resolution Advisory Detection}

In a similar way that a conflict detection algorithm checks whether or not a loss of separation is predicted to occur within a period of time, it is possible to design a resolution advisory detection algorithm that checks whether or not an RA is predicted to be issued for the ownship within a period of time. This sections presents an analytical formulation of such a resolution advisory detection algorithm, which is called RA3D.

RA3D is a function that takes as parameters the states of the ownship and intruder aircraft and a lookahead time interval, and returns a Boolean value that indicates whether an RA is predicted to be issued for the ownship within that time. It has been formally proved that, assuming accurate vector information and kinematic aircraft trajectories, RA3D correctly and completely characterizes the aircraft states that lead to an RA for the ownship within the lookahead time interval.

In order to define RA3D, it is necessary to first define a function that detects whether a horizontal RA will occur between two current or future times. This is accomplished by a function called RA2D $\mathrm{D}_{\ell}$ Then, the function RAZTimeInterval ${ }_{\ell}$ is defined that characterizes the time interval where the values of the functions Vertical_RA $A_{\ell}$ and $\mathrm{CD}_{2} \mathrm{D}_{\infty}$ are set to true. The function RA3D is defined by appropriately calling $\mathrm{RA}_{2} \mathrm{D}_{\ell}$ on the interval returned by RAZTimeInterval .

\section{IV.A. Characterization of Horizontal RAs}

As illustrated by Formula (12) in Section III, the value of $\tau_{\bmod \ell}(\mathbf{s}, \mathbf{v})$ determines whether there is a horizontal RA when the aircraft have horizontal relative position $\mathbf{s}$ and horizontal relative velocity $\mathbf{v}$. Thus, the minimum value of $\tau_{\bmod \ell}(\mathbf{s}+t \mathbf{v}, \mathbf{v})$, where $t$ ranges over the lookahead time interval $[B, T]$, determines whether or not a horizontal RA will occur for some time $t$ in this interval. The minimum value of $\tau_{\bmod \ell}$ can be computed with a concise formula, namely Formula (20), which defines the function Time_Min_TAUmod . $_{-}$This

\footnotetext{
${ }^{\mathrm{b}}$ Formula (19) assumes that the sensitivity level $\ell$ does not change in the time interval $[0, t]$.
} 
function computes exactly the time $t$ in $[B, T]$ at which $\tau_{\bmod \ell}(\mathbf{s}+t \mathbf{v}, \mathbf{v})$ attains its minimum value.

$$
\operatorname{Time}_{\text {Min_TAUmod }}(\mathbf{s}, \mathbf{v}, B, T) \equiv \begin{cases}B & \text { if }(\mathbf{s}+B \mathbf{v}) \cdot \mathbf{v} \geq 0 \\ t^{*}\left(\tau_{\min }\right) & \text { if } \Delta\left(\mathbf{s}, \mathbf{v}, \mathrm{DMOD}_{\ell}\right)<0 \\ T & \text { if }(\mathbf{s}+T \mathbf{v}) \cdot \mathbf{v}<0 \\ t^{*}(0) & \text { otherwise, }\end{cases}
$$

where

$$
\begin{aligned}
\tau_{\text {min }} & \equiv 2 \frac{\sqrt{-\Delta\left(\mathbf{s}, \mathbf{v}, \mathrm{DMOD}_{\ell}\right)}}{\|\mathbf{v}\|^{2}} \\
t^{*}(t) & \equiv \max \left(B, \min \left(T, t_{\mathrm{cpa}}(\mathbf{s}, \mathbf{v})-\frac{t}{2}\right)\right) .
\end{aligned}
$$

It should be noted that Formula (21) and Formula (22) are used in Formula (20) only in cases where the corresponding conditions imply that $\|\mathbf{v}\|^{2} \neq 0$ and $\Delta\left(\mathbf{s}, \mathbf{v}, \mathrm{DMOD}_{\ell}\right) \leq 0$. Hence, the function Time_Min_TAUmod $\ell$ is well-defined and does not involve divisions by zero or square roots of negative numbers. The function $\mathrm{RA}_{2} \mathrm{D}_{\ell}$ is defined as follows.

$$
\begin{aligned}
& \left.{\operatorname{RA} 2 D_{\ell}}_{\ell} \mathbf{s}, \mathbf{v}, B, T\right) \equiv\left(\Delta\left(\mathbf{s}, \mathbf{v}, \operatorname{DMOD}_{\ell}\right) \geq 0 \text { and } \mathbf{s}+B \mathbf{v}<0 \text { and } \mathbf{s}+T \mathbf{v} \geq 0\right) \text { or } \\
& \text { let } t=\text { Time_Min_TAUmod }(\mathbf{s}, \mathbf{v}, B, T) \text { in } \\
& \text { Horizontal_RA }(\mathbf{s}+t \mathbf{v}, \mathbf{v}) \text {. }
\end{aligned}
$$

The following proposition, which has been formally proved in the PVS theorem prover, asserts that, for a given sensitivity level $\ell$, the function $\mathrm{RA}_{2} \mathrm{D}_{\ell}$ characterizes the set of possible relative states that lead to a horizontal RA within a lookahead time interval.

Proposition 2. For all vectors $\mathbf{s}=\mathbf{s}_{o}-\mathbf{s}_{i}, \mathbf{v}=\mathbf{v}_{o}-\mathbf{v}_{i}$, and lookahead time interval $[B, T], R A 2 D_{\ell}(\mathbf{s}, \mathbf{v}, B, T)$ returns true if and only if there exists a time $t \in[B, T]$ where Horizontal_RA $A_{\ell}(\mathbf{s}+t \mathbf{v}, \mathbf{v})$ holds.

For some applications, it may be necessary to determine not only the existence of an RA within a lookahead time interval, but also the time interval when RAs are issued. The following function computes such a time interval for horizontal RAs.

$$
\begin{aligned}
& \text { RA2DTimeInterval }_{\ell}(\mathbf{s}, \mathbf{v}, B, T) \equiv \\
& \text { let } a=\|\mathbf{v}\|^{2} \text {, } \\
& b=2(\mathbf{s} \cdot \mathbf{v})+\mathrm{TAU}_{\ell}\|\mathbf{v}\|^{2}, \\
& c=\|\mathbf{s}\|^{2}+\operatorname{TAU}_{\ell}(\mathbf{s} \cdot \mathbf{v})-\mathrm{DMOD}_{\ell}^{2} \text { in } \\
& \text { if } a=0 \text { and }\|\mathbf{s}\| \leq \mathrm{DMOD}_{\ell} \text { then } \\
& {[B, T]} \\
& \text { else } \\
& \text { let } \theta=\Theta\left(\mathbf{s}, \mathbf{v}, \operatorname{DMOD}_{\ell}, 1\right) \text { in } \\
& \text { if }\|\mathbf{s}\| \leq \mathrm{DMOD}_{\ell} \text { then } \\
& {[B, \theta]} \\
& \text { elsif }\left(\mathbf{s} \cdot \mathbf{v} \geq 0 \text { or } b^{2}-4 a c<0\right. \text { then } \\
& {[T+1,0]} \\
& \text { elsif } \Delta\left(\mathbf{s}, \mathbf{v}, \operatorname{DMOD}_{\ell}\right) \geq 0 \text { then } \\
& {[\operatorname{root}(a, b, c,-1), \theta]} \\
& \text { else } \\
& {[\operatorname{root}(a, b, c,-1), \operatorname{root}(a, b, c, 1)]}
\end{aligned}
$$


The following proposition, which has been formally proved in the PVS theorem prover, asserts that, for a given sensitivity level $\ell$, the function RA2DTimeInterval $\ell$ computes a time interval that characterizes the times, within a lookahead time interval $[B, T]$, at which a horizontal RA will be issued.

Proposition 3. For all vectors $\mathbf{s}=\mathbf{s}_{o}-\mathbf{s}_{i}, \mathbf{v}=\mathbf{v}_{o}-\mathbf{v}_{i}$, and lookahead time interval $[B, T]$, if $R A 2 D T$ TimeInterval $l_{\ell}(\mathbf{s}, \mathbf{v}, B, T)$ returns the time interval $\left[t_{i n}, t_{\text {out }}\right]$, then for all times $t \in[B, T]$, Horizontal_RA $(\mathbf{s}+t \mathbf{v}, \mathbf{v})$ holds if and only if $t \in\left[t_{\text {in }}, t_{\text {out }}\right]$.

\section{IV.B. Characterization of RAs}

As noted above, the functions RA2D $\ell$ and RA2DTimeInterval $\ell$ can be used to, respectively, detect a horizontal RA within a lookahead time interval and compute the times, within the lookahead time interval, when a horizontal RA violation will occur. To completely formalize a TCAS II RA detection algorithm, functions RA3D and RA3DTimeInterval $l_{\ell}$, which are analogous to $\mathrm{RA} \mathrm{D}_{\ell}$ and RA2DTimeInterval $l_{\ell}$, are defined such that they take into account both the horizontal and vertical components of the TCAS II RA logic. These functions have as parameters the state information of the ownship, i.e., $\mathbf{s}_{o}, s_{o z}, \mathbf{v}_{o}, v_{o z}$, the state information of the intruder aircraft, i.e., $\mathbf{s}_{i}, s_{i z}, \mathbf{v}_{i}, v_{i z}$, the lookahead time interval $[B, T]$, and a flag hmdf? that indicates whether or not the horizontal miss distance filter should be used. For RA detection according to the TCAS II RA logic, that flag should always be set to true.

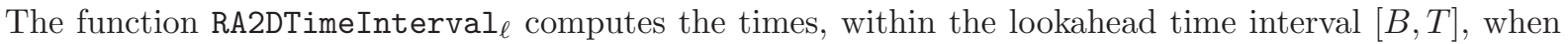
an RA violation will occur. It is formally defined as follows.

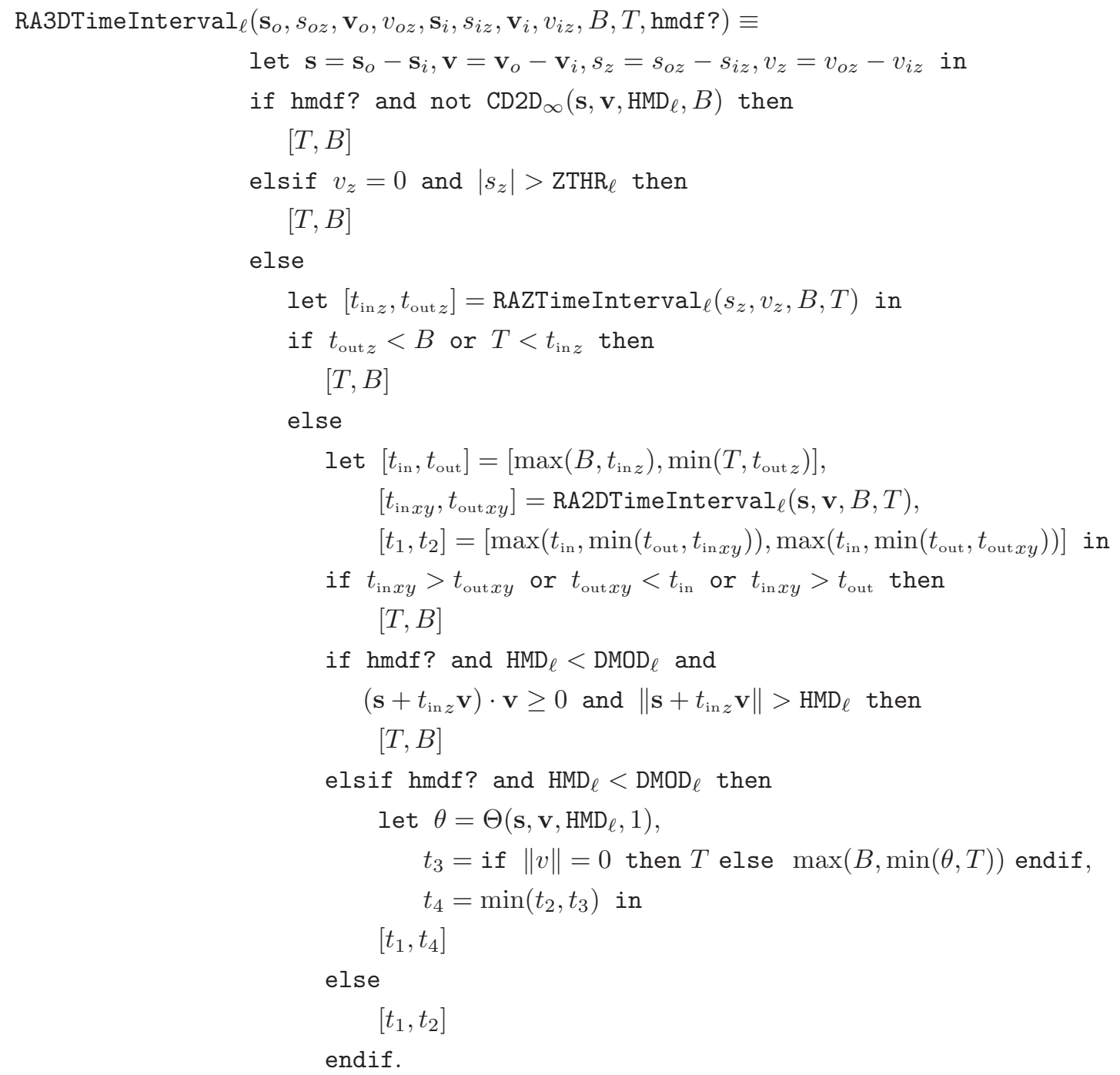


The function RAZTimeInterval $\ell$ used in Formula (25) computes the time interval when vertical separation with respect to $\mathrm{ZTHR}_{\ell}$ will be lost within the time interval $[B, T]$. It is defined by Formula (26).

$$
\text { RAZTimeInterval } \ell_{\ell}\left(s_{z}, v_{z}, B, T\right) \equiv \begin{cases}{[B, T]} & \text { if } v_{z}=0, \\ \left.\left[\frac{-\operatorname{sign}\left(v_{z}\right) H}{v_{z}}, \frac{\operatorname{sign}\left(v_{z}\right) H}{v_{z}}\right)\right] & \text { otherwise, }\end{cases}
$$

where $H \equiv \max \left(\mathrm{ZTHR}_{\ell}, \mathrm{TAU}_{\ell}\left|v_{z}\right|\right)$ and $\operatorname{sign}\left(v_{z}\right)$ denotes the sign of $v_{z}$, i.e., -1 when $v_{z}<0$ and 1 when $v_{z}>0$.

The function RA3D detects RAs by checking the time interval that is returned by RA3DTimeInterval ${ }_{\ell}$, where $\ell$ is the sensitivity level corresponding to $s_{o z}$.

$$
\begin{aligned}
& \operatorname{RA3D}\left(\mathbf{s}_{o}, s_{o z}, \mathbf{v}_{o}, v_{o z}, \mathbf{s}_{i}, s_{i z}, \mathbf{v}_{i}, v_{i z}, B, T\right) \equiv \\
& \quad \text { let }\left[t_{\text {in }}, t_{\text {out }}\right]=\text { RA3DTimeInterval } \ell_{\ell}\left(\mathbf{s}_{o}, s_{o z}, \mathbf{v}_{o}, v_{o z}, \mathbf{s}_{i}, s_{i z}, \mathbf{v}_{i}, v_{i z}, B, T, \text { true }\right) \text { in } \\
& \quad t_{\text {in }} \leq t_{\text {out }} .
\end{aligned}
$$

Just as for the rest the mathematical development presented in this paper, the following proposition has been formally proved in the PVS theorem prover. It states that the function RA3D characterizes the set of aircraft states that will lead to an RA for the ownship within a lookahead time interval assuming that the aircraft follow linear projections of their current states and the sensitivity level remains constant.

Proposition 4. For all ownship states $\mathbf{s}_{o}, s_{o z}, \mathbf{v}_{o}, v_{o z}$, intruder states $\mathbf{s}_{i}, s_{i z}, \mathbf{v}_{i}, v_{i z}$, and lookahead time interval $[B, T], R A 3 D\left(\mathbf{s}_{o}, s_{o z}, \mathbf{v}_{o}, v_{o z}, \mathbf{s}_{i}, s_{i z}, \mathbf{v}_{i}, v_{i z}, B, T\right)$ returns true if and only if there exists a $t \in[B, T]$ where TCASII_RA_at $\left(\mathbf{s}_{o}, s_{o z}, \mathbf{v}_{o}, v_{o z}, \mathbf{s}_{i}, s_{i z}, \mathbf{v}_{i}, v_{i z}, t\right)$ holds.

\section{Corrective Resolution Advisories}

As explained in Section II, there are two types of resolution advisories: corrective and preventive. Corrective RAs require a trajectory change by the ownship to regain a minimum altitude limit (ALIM), whose value depends on the sensitivity level. This section presents mathematical formulas that determine if a given $\mathrm{RA}$ is either corrective or preventive.

The function sep_at, defined by Formula (28), predicts the vertical separation between the aircraft at a given time $t$ assuming a target vertical speed $v$ for the ownship. The ownship is assumed to fly at constant ground speed and constant vertical acceleration $a$. Once the target vertical speed $v$ is reached the ownship continues to fly at constant vertical speed. The function own_alt_at, defined by Formula (29), computes the vertical altitude of the ownship at time $t$ given a target vertical speed $v$ and acceleration $a$. The parameter $\epsilon$ specifies a possible direction for the vertical ownship maneuver, which is upward when $\epsilon=1$ and downward when $\epsilon=-1$. The intruder is assumed to continue its trajectory at its current vertical speed.

$$
\begin{aligned}
& \operatorname{sep} \_a t\left(s_{o z}, v_{o z}, s_{i z}, v_{i z}, v, a, \epsilon, t\right) \equiv \\
& \text { let } o=\text { own_alt_at }\left(s_{o z}, v_{o z},|v|, a, \epsilon \operatorname{sign}(v), t\right), \\
& i=s_{i z}+t v_{i z} \text { in } \\
& \epsilon(o-i) .
\end{aligned}
$$

$$
\begin{aligned}
& \text { own_alt_at }\left(s_{o z}, v_{o z}, v, a, \epsilon, t\right) \equiv \\
& \text { let } s=\operatorname{stop} \_ \text {accel }\left(v_{o z}, v, a, \epsilon, t\right) \text {, } \\
& q=\min (t, s), \\
& l=\max (0, t-s) \text { in } \\
& \epsilon q^{2} \frac{a}{2}+q v_{o z}+s_{o z}+\epsilon l v \text {. }
\end{aligned}
$$

The function stop_accel computes the time at which the ownship reaches the target vertical speed $v$. It is 
defined as follows.

$$
\begin{aligned}
& \text { stop_accel }\left(v_{o z}, v, a, \epsilon, t\right) \equiv \\
& \text { if } t \leq 0 \text { or } \epsilon v_{o z} \geq v \text { then } 0 \\
& \text { else } \frac{\epsilon v-v_{o z}}{\epsilon a} \\
& \text { endif. }
\end{aligned}
$$

The sense of an RA is computed based on the direction for the ownship maneuver that provides a greater vertical separation, with a bias towards the non-crossing direction. The function RA_sense computes such a direction, where $\mathrm{ALIM}_{\ell}$ is the altitude limit for a given sensitivity level $\ell$.

$$
\begin{aligned}
& \text { RA_sense }\left(s_{o z}, v_{o z}, s_{i z}, v_{i z}, v, a, t\right) \equiv \\
& \text { let } o_{\uparrow}=\text { own_alt_at }\left(s_{o z}, v_{o z}, v, a, 1, t\right), \\
& \quad o_{\downarrow}=\text { own_alt_at }\left(s_{o z}, v_{o z}, v, a,-1, t\right), \\
& i=s_{i z}+t v_{i z}, \\
& u=o_{\uparrow}-i, \\
& \quad d=i-o_{\downarrow} \text { in } \\
& \text { if } \operatorname{sign}\left(s_{o z}-s_{i z}\right)=1 \text { and } u \geq \operatorname{ALIM}_{\ell} \text { then } 1 \\
& \text { elsif } \operatorname{sign}\left(s_{o z}-s_{i z}\right)=-1 \text { and } d \geq \operatorname{ALIM}_{\ell} \text { then }-1 \\
& \text { elsif } u \geq d \text { then } \\
& \text { else }-1 \\
& \text { endif. }
\end{aligned}
$$

An RA is corrective if the altitude limit is not cleared when the ownship maneuvers in the direction of the RA sense. The Boolean function corrective specifies an algorithm that returns true when a given RA is corrective.

$$
\begin{aligned}
& \operatorname{corrective}\left(\mathbf{s}_{o}, s_{o z}, \mathbf{v}_{o}, v_{o z}, \mathbf{s}_{i}, s_{i z}, \mathbf{v}_{i}, v_{i z}, v, a\right) \equiv \\
& \text { let } \mathbf{s}=\mathbf{s}_{o}-\mathbf{s}_{i}, \mathbf{v}=\mathbf{v}_{o}-\mathbf{v}_{i}, s_{z}=s_{o z}-s_{i z}, v_{z}=v_{o z}-v_{i z}, \\
& t=\tau_{\bmod \ell}(\mathbf{s}, \mathbf{v}) \\
& \epsilon=\operatorname{RA}_{-} \operatorname{sense}\left(s_{o z}, v_{o z}, s_{i z}, v_{i z}, v, a, t\right) \text { in } \\
& \|\mathbf{s}\|<\operatorname{DMOD}_{\ell} \text { or } \\
& \left(\mathbf{s} \cdot \mathbf{v}<0 \text { and } \epsilon\left(s_{z}+t v_{z}\right)<\operatorname{ALIM}_{\ell}\right),
\end{aligned}
$$

where $\ell$ is the sensitivity level corresponding to $s_{o z}$.

\section{Related Work}

Formal models of TCAS have been proposed before. Leveson et al. provides a complete specification of the TCAS II logic in a tabular notation called Requirements State Machine Language (RSML) [8]. Livadas et al. presents a high level model of the core components of TCAS using the formalism of Hybrid Input/Output Automata (HIOA) [9]. The model presented here is more modest than those models. This paper focuses on the collision avoidance logic that deals with resolution advisories and assumes that accurate state vector information will be available to sense and avoid systems. A key difference between this work and other models, such as those listed above, is the introduction of this vector information, which enables the derivations of the concise formulas presented in this paper for computing TCAS II RA information, especially those formulas used to analytically detect future RAs. The assumption that accurate state vector information is available reduces the complexity in the TCAS II logic concerning aircraft tracking and enables the analytical presentation, which is based on vector arithmetic. It should be noted that the algorithm presented in Section V projects aircraft vertical trajectories using constant acceleration and a target vertical speed as specified in the TCAS II logic, rather than constant vertical speed as specified in [9].

Safety properties related to issuance of resolution advisories have been verified by Coen-Porisini et al. [10] using symbolic execution and, more recently, by Gotlieb [11] using constraint programming. These works rely 
on a C implementation of the core TCAS II RA logic that is available at the Software-artifact Infrastructure Repository (SIR) maintained by the University of Nebraska-Lincoln. That code deals with high-level aspects of the function that computes the sense of an RA, but does not implement the mathematical formulas that determine whether or not an RA is issued.

\section{Conclusion}

This paper presents a formal development that consists of a mathematical model of the TCAS II resolution advisory logic and algorithms for detecting RAs within a given lookahead time interval and for checking whether a given RA is corrective or not.

As far as the authors know, an algorithm for detecting resolution advisories has not been proposed before. This algorithm is a key component of NASA's Separation Assurance concept for the integration of UAS in the NAS [4]. In particular, it is used to implement airborne and ground capabilities that allow UAS pilots to avoid encounter scenarios that are not well-clear vis-a-vis systems such as TCAS. It should be noted that the algorithm presented here can be easily parameterized into an algorithm for detecting traffic advisories. This is possible since the TCAS II logic for traffic advisories and the logic for resolution advisories mainly differ in the values of the time and distance threshold parameters and the use of an horizontal miss distance filter. Indeed, the function TA3D defined by Formula (33) detects Traffic Alerts (TAs) within a lookahead time interval $[B, T]$, when the threshold values $\mathrm{TAU}_{\ell}, \mathrm{DMOD}_{\ell}$, and $\mathrm{ZTHR}_{\ell}$ are taken from Table $2[2]$. In this case, the function RA3DTimeInterval $l_{\ell}$ is called with the parameter corresponding to the horizontal miss distance filter hmdf? set to false.

$$
\begin{aligned}
& \operatorname{TA3D}\left(\mathbf{s}_{o}, s_{o z}, \mathbf{v}_{o}, v_{o z}, \mathbf{s}_{i}, s_{i z}, \mathbf{v}_{i}, v_{i z}, B, T\right) \equiv \\
& \operatorname{let~}\left[t_{\text {in }}, t_{\text {out }}\right]=\text { RA3DTimeInterval } \\
& \quad\left(\mathbf{s}_{o}, s_{o z}, \mathbf{v}_{o}, v_{o z}, \mathbf{s}_{i}, s_{i z}, \mathbf{v}_{i}, v_{i z}, B, T, \text { false }\right) \text { in } \\
& \quad t_{\text {in }} \leq t_{\text {out }} .
\end{aligned}
$$

Table 2. TCAS Sensitivity Level Definition and Alarm Thresholds for TAs

\begin{tabular}{|c|c|c|c|c|}
\hline $\begin{array}{c}\text { Ownship Altitude } \\
\text { (feet) }\end{array}$ & SL & $\begin{array}{c}\text { TAU } \\
\text { (sec) }\end{array}$ & $\begin{array}{c}\text { DMOD } \\
\text { (nmi) }\end{array}$ & $\begin{array}{c}\text { ZTHR } \\
\text { (feet) }\end{array}$ \\
\hline $\mathbf{1 0 0 0 - \mathbf { 2 3 5 0 }}$ & 2 & 20 & 0.30 & 850 \\
\hline $\mathbf{1 0 0 0}-\mathbf{2 3 5 0}$ & 3 & 25 & 0.33 & 850 \\
\hline $\mathbf{2 3 5 0}-\mathbf{5 0 0 0}$ & 4 & 30 & 0.48 & 850 \\
\hline $\mathbf{5 0 0 0}-\mathbf{1 0 0 0 0}$ & 5 & 40 & 0.75 & 850 \\
\hline $\mathbf{1 0 0 0 0}-\mathbf{2 0 0 0 0}$ & 6 & 45 & 1.0 & 850 \\
\hline $\mathbf{2 0 0 0 0}-\mathbf{4 2 0 0 0}$ & 7 & 48 & 1.3 & 850 \\
\hline $\mathbf{4 2 0 0 0}$ & 7 & 48 & 1.3 & 1200 \\
\hline
\end{tabular}

Finally, it is emphasized that the mathematical development presented here has been formalized and mechanically verified in PVS. This level of rigor is justified by the safety-critical role that sense-and-avoid UAS systems may play in the future airspace system.

\section{References} 2009.

${ }^{1}$ FAA Sponsored Sense and Avoid Workshop, "Sense and avoid (SAA) for unmanned aircraft systems (UAS)," October

${ }^{2}$ U.S. Department of Transportation Federal Aviation Administration, "Introduction to TCAS II Version 7.1," February 2011.

${ }^{3}$ RTCA SC-147, "RTCA-DO-185B, Minimum operational performance standards for traffic alert and collision avoidance system II (TCAS II)," July 2009.

${ }^{4}$ Consiglio, M., Chamberlain, J., Muñoz, C., and Hoffler, K., "Concept of integration for UAS operations in the NAS," Proceedings of 28th International Congress of the Aeronautical Sciences, ICAS 2012, Brisbane, Australia, 2012.

${ }^{5}$ Owre, S., Rushby, J., and Shankar, N., "PVS: A Prototype Verification System," Proc. 11th Int. Conf. on Automated Deduction, edited by D. Kapur, Vol. 607 of Lecture Notes in Artificial Intelligence, Springer-Verlag, June 1992, pp. 748-752. 
${ }^{6}$ Kuchar, J., "Update on the analysis of ACAS performance on Global Hawk," May 2006, ASP/WG A/WP A10-04.

${ }^{7}$ Hammer, J., "Horizontal miss distance filter system for suppressing false resolution alerts," October 1996, U.S. Patent $5,566,074$.

${ }^{8}$ Leveson, N. G., Heimdahl, M., Hildreth, H., and Reese, J. D., "Requirements Specification for Process-Control Systems," IEEE Transactions on Software Engineering, Vol. 20, No. 9, 1994, pp. 684-707.

${ }^{9}$ Livadas, C., Lygeros, J., and Lynch, N., "High-Level Modeling and Analysis of the Traffic Alert and Collision Avoidance System (TCAS)," Proceedings of IEEE, Special Issue on Hybrid Systems: Theory \& Applications, Vol. 88, No. 7, July 2000, pp. 926-948.

${ }^{10}$ Coen-Porisini, A., Denaro, G., Ghezzi, C., and Pezzè, M., "Using Symbolic Execution for Verifying Safety-Critical Systems," Proceedings of the European Software Engineering Conference (ESEC 2001/FSE 9), No. 142-151, ACM, Vienna, Austria, 2001.

${ }^{11}$ Gotlieb, A., "TCAS software verification using constraint programming," The Knowledge Engineering Review, Vol. 27, No. 3, September 2012, pp. 343-360. 